\title{
GeAs: Highly Anisotropic van der Waals Thermoelectric Material
}

Kathleen Lee, ${ }^{\S}$ Saeed Kamali, ${ }^{\#}$ Tore Ericsson, ${ }^{\&}$ Maverick Bellard, ${ }^{\S}$ Kirill Kovnir ${ }^{\S} *$

${ }^{\S}$ Department of Chemistry, University of California, Davis, One Shields Avenue, Davis, CA 95616

"Mechanical, Aerospace \& Biomedical Engineering Department, University of Tennessee Space Institute,

Tullahoma, TN 37388

${ }^{\&}$ Department of Chemistry - Ångström Laboratory, Uppsala University, Box 538, SE-75121 Uppsala, Sweden

\section{Supporting Information}

\section{Table of Contents}

Table S1. Selected bond distances in GeAs and Sn-doped GeAs ............................................................. 22

Table S2. Atomic coordinates and equivalent displacement parameters for GeAs and Sn-doped GeAs ....S2

Figure S1. Powder XRD patterns of sintered pellets of GeAs and Sn-doped GeAs ...................................S3

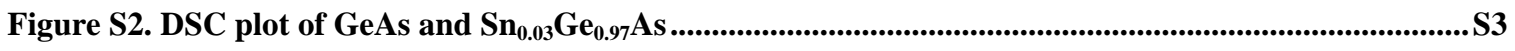

Figure S3. EDX spectra of Sn-doped GeAs and pure GeAs ..........................................................................S4

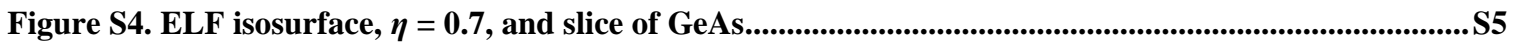

Figure S5. Lattice and electronic contribution to the total thermal conductivity for GeAs ...........................S5 
Table S1. Selected bond distances in GeAs and Sn-doped GeAs.

\begin{tabular}{|l|l|l|}
\hline Atoms & \multicolumn{1}{|c|}{$\mathrm{GeAs}$} & $\mathrm{Sn}_{0.08(2)} \mathrm{Ge}_{0.92} \mathrm{As}^{a}$ \\
\hline Ge1-Ge2 & $2.451(1)$ & $2.54(2)$ \\
\hline Ge3-Ge3 & $2.464(1)$ & $2.461(5)$ \\
\hline Ge1-As1 & $2.452(1)$ & $2.41(1)$ \\
\hline Ge1-As3 & $2.4654(6) \times 2$ & $2.451(5) \times 2$ \\
\hline Ge2-As1 & $2.4567(7) \times 2$ & $2.481(3) \times 2$ \\
\hline Ge2-As2 & $2.4494(4)$ & $2.458(3)$ \\
\hline Ge3-As2 & $2.4711(7) \times 2$ & $2.505(4) \times 2$ \\
\hline Ge3-As3 & $2.490(1)$ & $2.472(5)$ \\
\hline As1-As2 & $2.359(1)$ & $3.365(5)$ \\
\hline As1-As3 & $3.4320(9) \times 2$ & $3.446(4) \times 2$ \\
\hline As2-As2 & $3.608(1) \times 2$ & $3.615(4) \times 2$ \\
\hline As3-As3 & $3.501(1)$ & $3.515(5)$ \\
\hline Ge11-Ge2 & -- & $2.32(2)$ \\
\hline Sn1-Sn21 & -- & $2.91(3)$ \\
\hline Sn31-Sn31 & -- & $2.75(3)$ \\
\hline
\end{tabular}

${ }^{a}$ Mainly distances comparable with those in GeAs are shown for the Sn-containing compound.

Table S2. Atomic coordinates and equivalent displacement parameters for GeAs and Sn-doped GeAs.

\begin{tabular}{|c|c|c|c|c|c|c|}
\hline \multicolumn{7}{|c|}{ GeAs } \\
\hline Atom & Wyckoff & $x / a$ & $y / b$ & $z / c$ & s.o.f. & $U_{\text {eq }}$ or $U_{\text {iso }}$ \\
\hline $\mathrm{Ge}(1)$ & $4 i$ & $0.06832(4)$ & 0 & $0.08986(7)$ & 1 & $0.0067(1)$ \\
\hline $\mathrm{Ge}(2)$ & $4 i$ & $0.62725(4)$ & 0 & $0.42405(3)$ & 1 & $0.0068(1)$ \\
\hline $\mathrm{Ge}(3)$ & $4 i$ & $0.74483(4)$ & 0 & $0.28662(3)$ & 1 & $0.0070(1)$ \\
\hline As(1) & $4 i$ & $0.15340(4)$ & 0 & $0.68667(6)$ & 1 & $0.0070(1)$ \\
\hline As(2) & $4 i$ & $0.15984(4)$ & 0 & $0.04165(6)$ & 1 & $0.0068(1)$ \\
\hline As(3) & $4 i$ & $0.53166(4)$ & 0 & $0.33322(7)$ & 1 & $0.0069(1)$ \\
\hline \multicolumn{7}{|c|}{$\mathrm{Sn}_{0.08(2)} \mathrm{Ge}_{0.92} \mathrm{As}$} \\
\hline $\mathrm{Ge}(1)$ & $4 i$ & $0.1255(8)$ & 0 & $0.430(1)$ & $0.47(8)$ & $0.0105(6)$ \\
\hline $\operatorname{Sn}(1)$ & $4 i$ & $0.1255(8)$ & 0 & $0.430(1)$ & $0.07(3)$ & $0.0105(6)$ \\
\hline $\mathrm{Ge}(11)$ & $4 i$ & $0.133(1)$ & 0 & $0.417(1)$ & $0.46(7)$ & $0.0105(6)$ \\
\hline $\mathrm{Ge}(2)$ & $4 i$ & $0.2437(2)$ & 0 & $0.2877(3)$ & $0.92(1)$ & $0.0115(4)$ \\
\hline $\operatorname{Sn}(21)$ & $4 i$ & $0.262(2)$ & 0 & $0.269(2)$ & 0.08 & $0.0115(4)$ \\
\hline $\mathrm{Ge}(3)$ & $4 i$ & $0.5669(2)$ & 0 & $0.0917(3)$ & $0.91(1)$ & $0.0114(4)$ \\
\hline $\operatorname{Sn}(31)$ & $4 i$ & $0.584(1)$ & 0 & $0.075(2)$ & 0.09 & $0.0114(4)$ \\
\hline As(1) & $4 i$ & $0.1532(1)$ & 0 & $0.6872(2)$ & 1 & $0.0162(4)$ \\
\hline $\operatorname{As}(2)$ & $4 i$ & $0.1603(1)$ & 0 & $0.0417(2)$ & 1 & $0.0160(4)$ \\
\hline As(3) & $4 i$ & $0.5317(1)$ & 0 & $0.3332(2)$ & 1 & $0.0162(4)$ \\
\hline
\end{tabular}




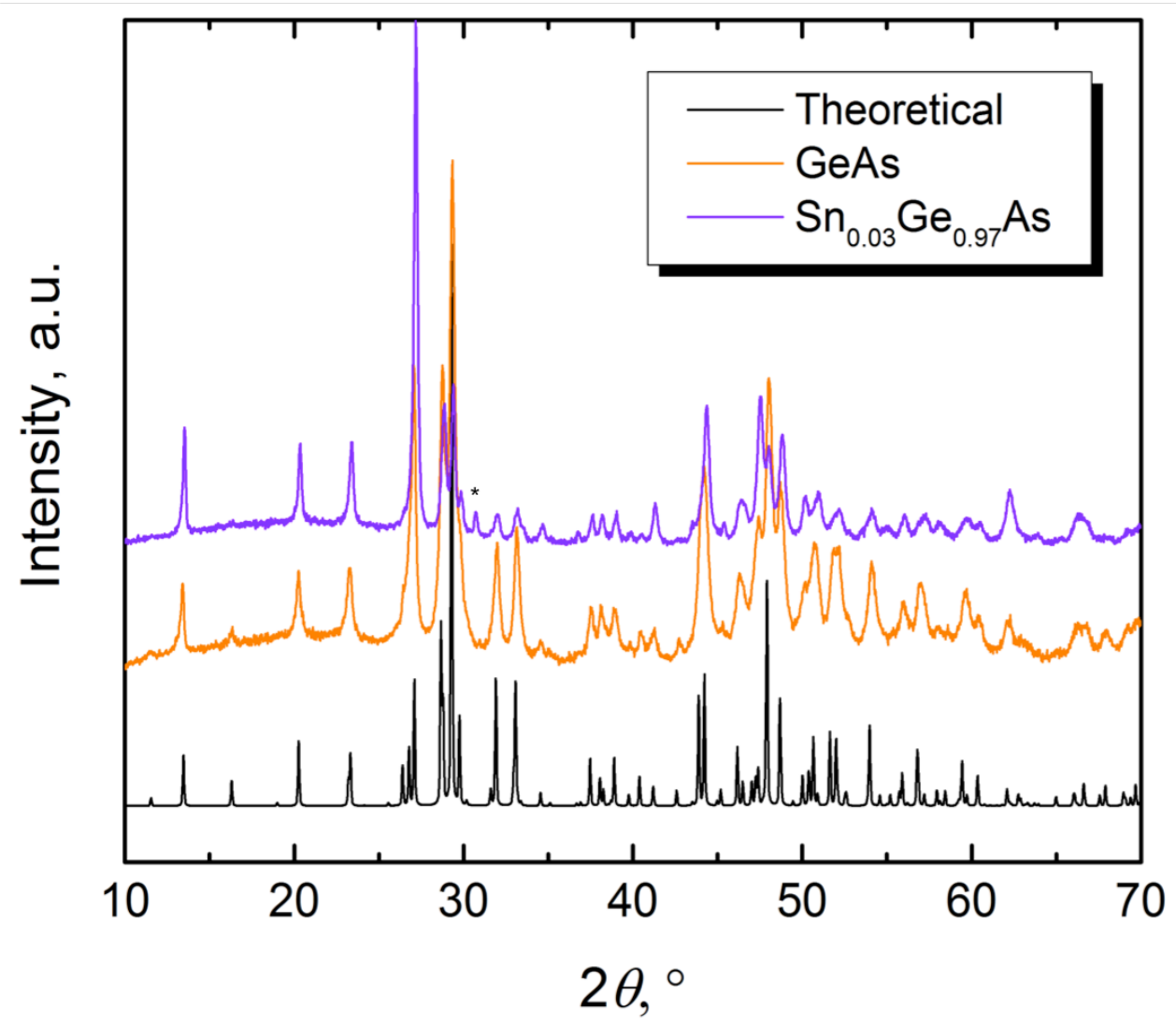

Figure S1. Powder XRD patterns of sintered pellets of GeAs and Sn-doped GeAs. Shifts in the patterns are due to the varying heights of the pellets. An unknown impurity in the $\mathrm{Sn}_{0.03} \mathrm{Ge}_{0.97} \mathrm{As}$ sample is indicated by an asterisk.
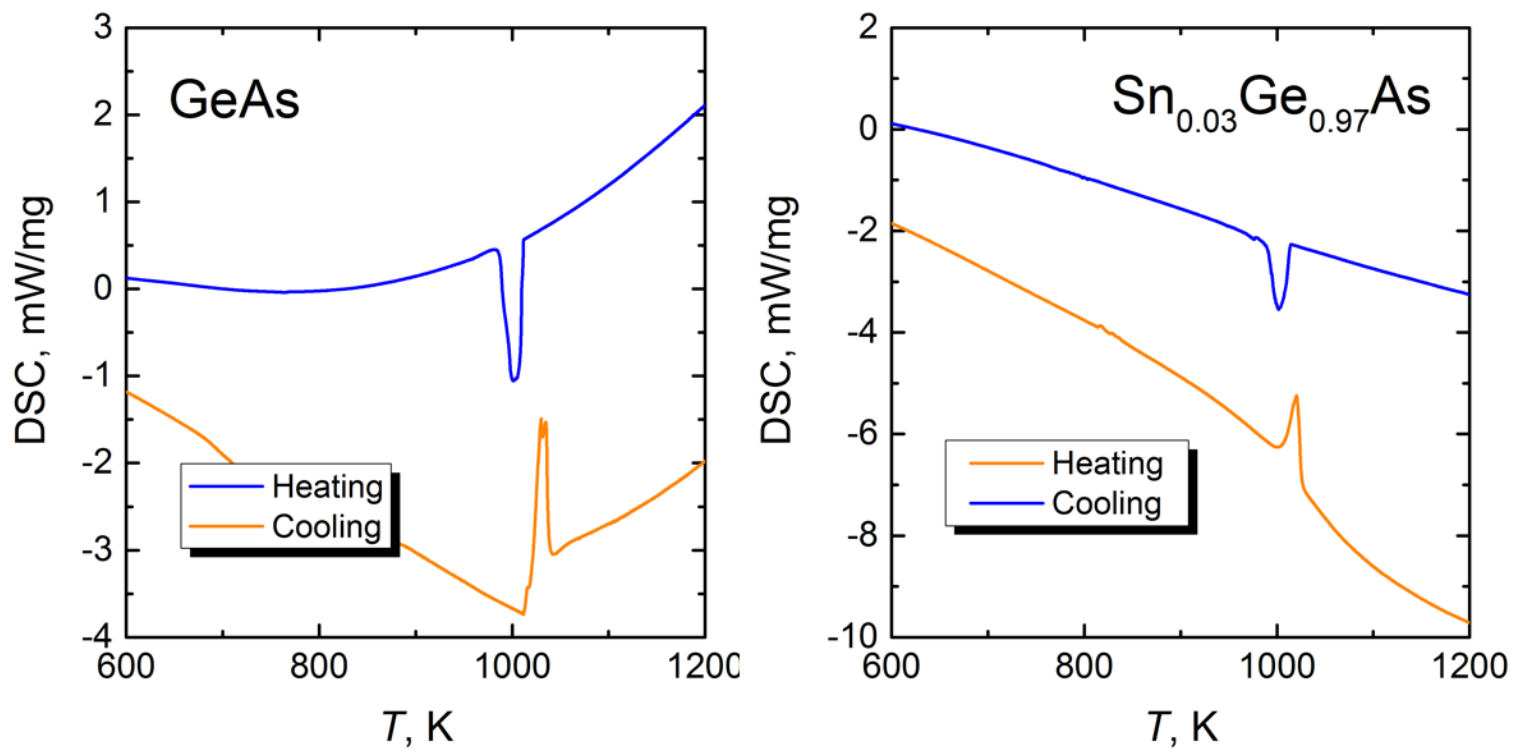

Figure S2. DSC plot of GeAs and $\mathrm{Sn}_{0.03} \mathrm{Ge}_{0.97} \mathrm{As}$. 


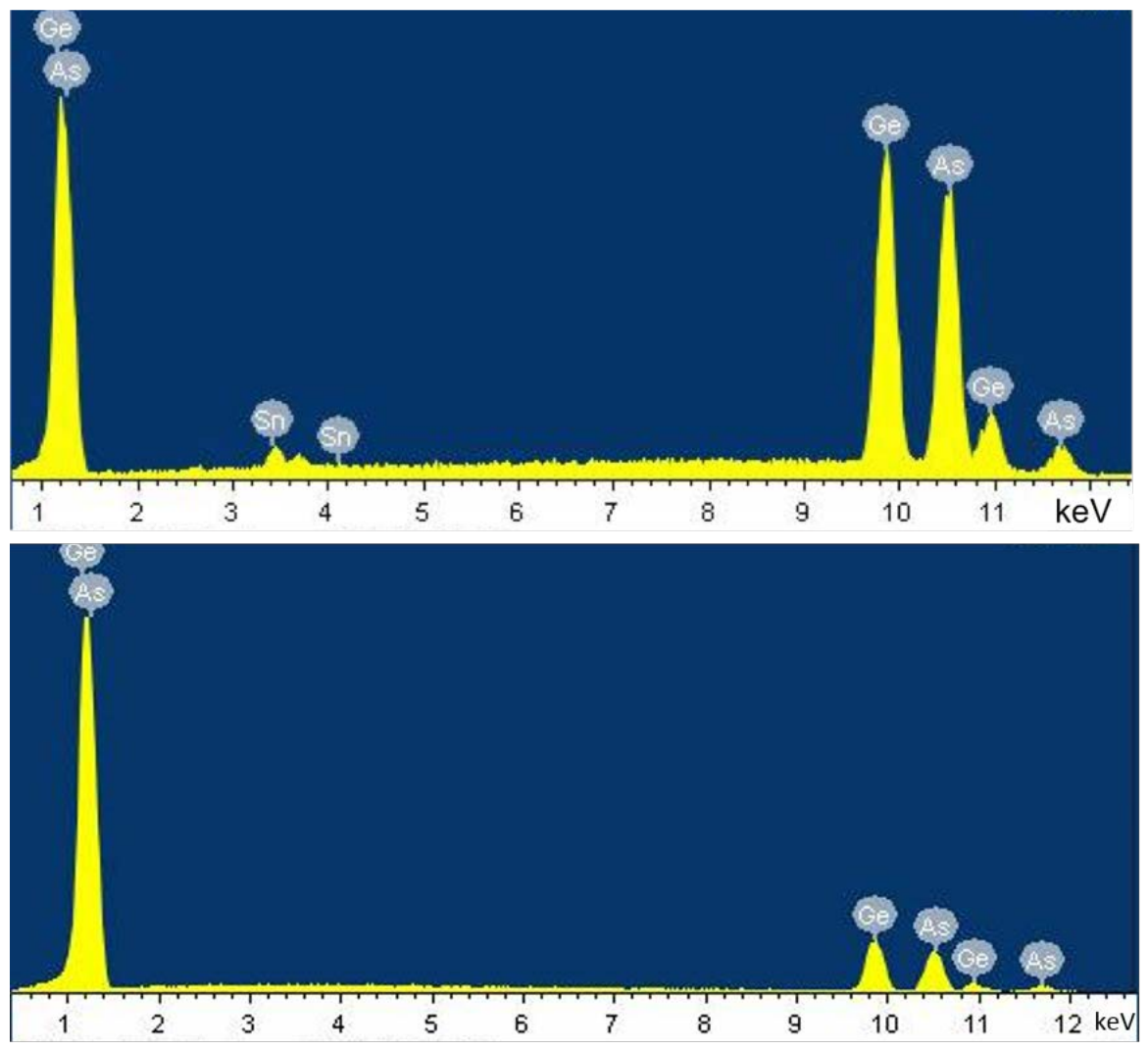

Figure S3. EDX spectra of (top) Sn-doped GeAs and (bottom) pure GeAs. 

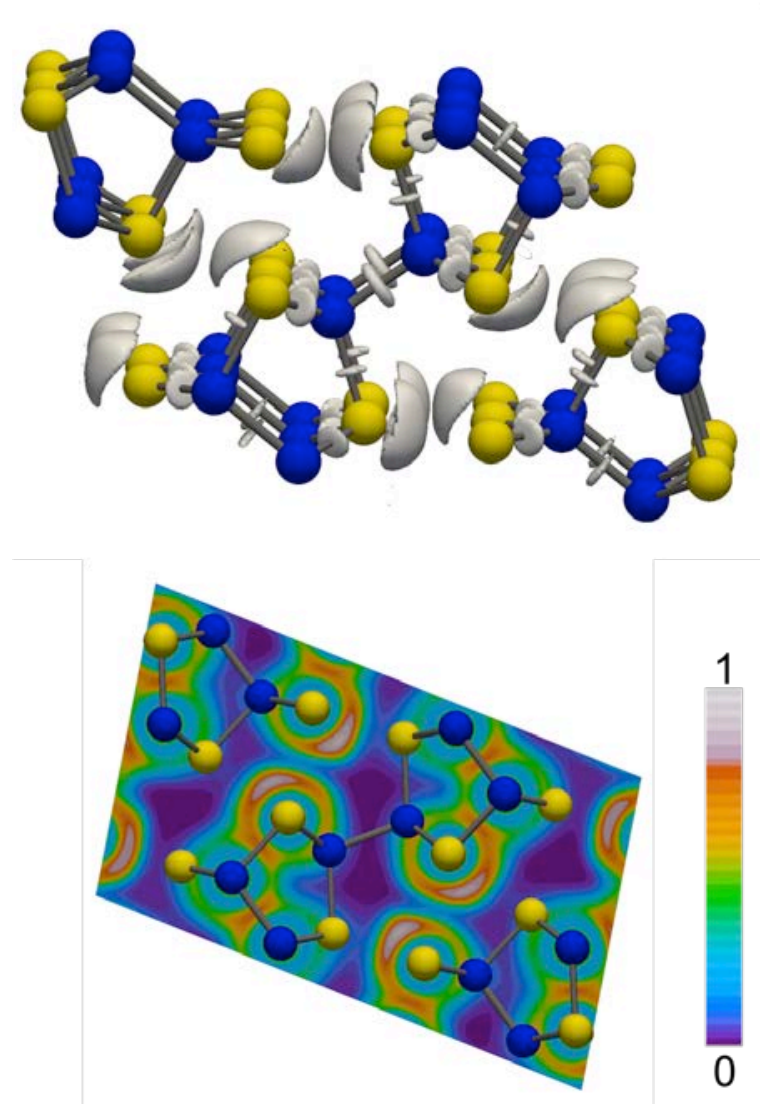

Figure S4. ELF isosurface, $\eta=0.7$ (top), and slice (bottom) of GeAs. Ge: blue, As: yellow. Scalebar shown on bottom right.
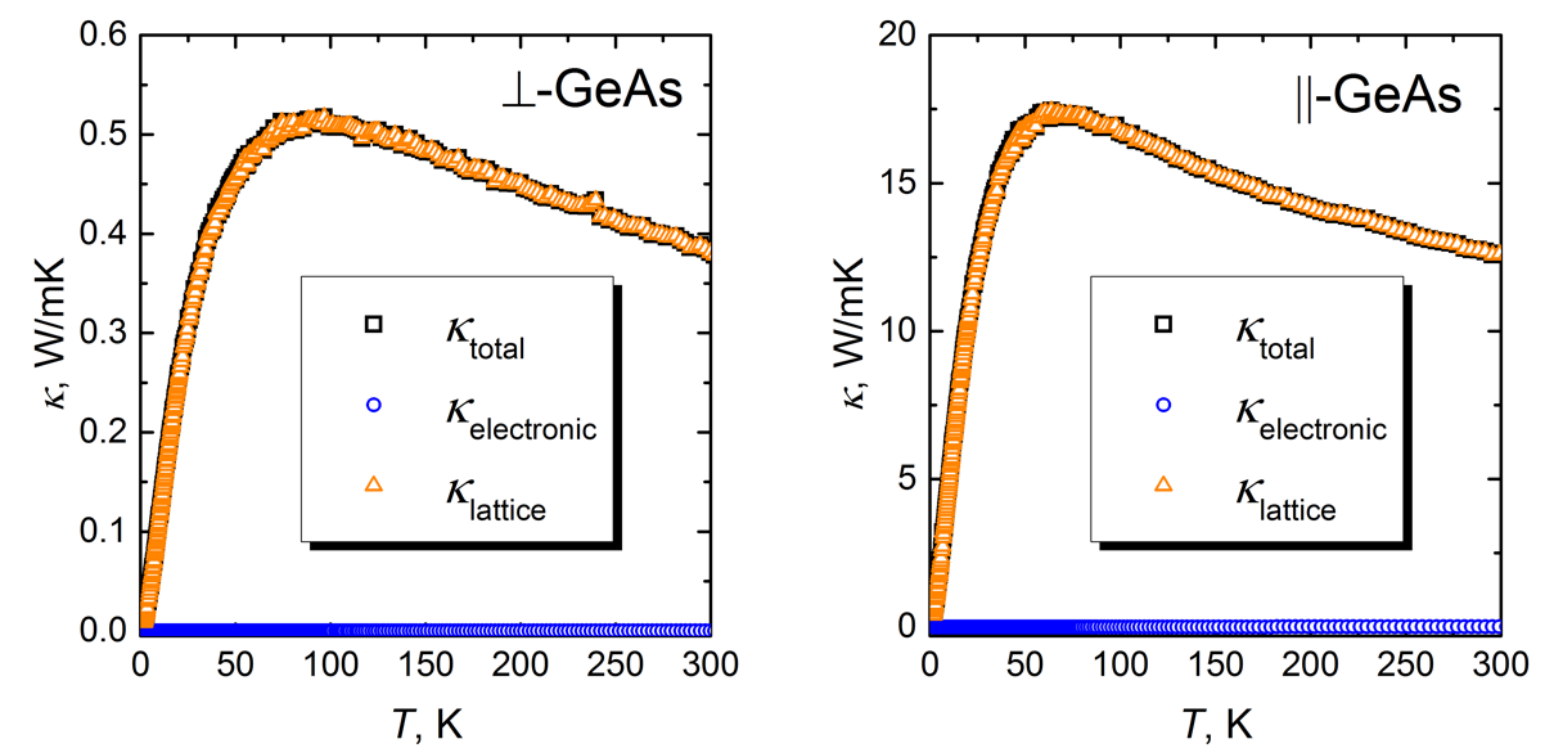

Figure S5. Lattice and electronic contribution to the total thermal conductivity for GeAs. Note that the black and orange data points essentially overlap. 